\title{
les lieux d'Hécate
}

\author{
Nadine Ltaif
}

\section{Hecate's Domain}

May 6

I had sunk my whole life in that island. Island? A mound of bones. Solitude, my safety. Island of serpent to welcome history, but which one? Mine? Yes, but transposed, displaced. By magic, or faith? Where is the witch or serpent lady, my fairy, my Eve who brought me into the world? Let her keep silent about all the love I've given her ...

May 15

Worry about my future behaviour after the flame of rage has died down. Dangerous, a flame. Keep quiet and pray, anti-semitism in France as upsetting as the bombing of Lebanon.

I don't know what makes me write again and again. If I abandon it, it comes again suddenly, at any rate, in all ways, there is a rhythm or breath that comes to life of its own accord sometimes, that takes flight ... becomes birds, has a bird's eye view of the landscape. Sun to bake my face, my mother's hidden under the shadow.

May 25

Terrible moody day. Abominal oriental dependency on family. Difficult to see what's happening in me... Not yet nine and the day's going to be long

6 mai 1990

J'avais échoué ma vie entière dans cette île. Une île, un monticule d'ossements. La Solitude véritable, inévitable, seule loi à mon secours, ma sauvegarde, mon sauvetage,

L'île du serpent, pour accueillir l'histoire, laquelle? La mienne? Oui. Mais transposée. Déplacée, Comme on déplace une montagne. Tour de Magie ou Foi?

Où est la sorcière, où la dame avec une queue de serpent. Où est ma fée, ma Eve qui m'a mise au monde, 
Ou ... celle qui m'enfante, une Nième fois. Où est-elle, celle avec qui j'ai échangé des secrets

Qu'elle garde le silence sur tout l'amour que je lui ai manifesté. Pour elle toutes mes peines d'amour. Tout mes plaisirs aussi (pour elle); les lacs pour moi sont toujours des étendues de larmes.

15 mai

Beaucoup de préoccupations au sujet de mon comportement futur. Comment agir avec celle qui a été la plus importante figure dans ma vie émotive à Montréal. Maintenant que la fureur irrationnelle s'est calmée. Que reste-t-il de cette flamme?

Ah que c'est dangereux une flamme, une braise, prête à se ranimer à n'importe quel moment de manière imprévu,

Je dois garder le silence et prier, anti-sémitisme en France et je suis bouleversée par cet événement comme par les bombardements qui tourmentent le Liban des peintures de Goya

je ne sais pas ce qui me fait écrire encore et encore, si je dois laisser reposer

car ça revient seul et d'un coup

de toute manière

et de toutes les manières

il y a le rythme ou le souffle qui se ranime parfois

De lui-même

Qui prend l'envol

Se déploie et traverse les continents

fend l'air

comme une aile

Et va se poser sur un arbre

Une branche d'arbre nu dénudé de l'hiver

Ce matin sur le lac, avec les marcheurs tout jeunes de «Moscou à Jérusalem», j'ai marché avec ma mère, ne prenant pas la même route

Nous regardions vers l'ouest, notre inévitable marche

Des hirondelles victorieuses étaient revenues sur la surface du lac 
Rasant l'eau, la tête et le manteau teintés

de bleu luisant seulement par reflet

j'ai vu une corneille

Et un jeune étourneau

j'ai entendu la voix de la corneille

et je l'ai différencié de celle

du corbeau

j'ai vu des arbres

plantés profondément dans le

sol canadien

j'ai vu le ciel bleu, une peinture de Pissaro j'ai vu.

Les eaux du lac artificiel,

Et le soleil dans un abri du vent

pour cuire mon visage

celui de ma mère abrité sous un ombrage

25 mai

Journée d'humeur exécrable. Abominable dépendance orientale à la famille. Extrêmement difficile de voir clairement ce qui se passe en moi. Vains espoirs. Dépendence encore là où il n'y a rien. Ni amour ni attache

Rien qu'illusion, immense désespoir, rien ne se trace devant moi, Il $\mathrm{n}^{\prime} \mathrm{y}$ a pas de route déjà tracée

Pas de chemin parsemé de cailloux. Le petit Poucet m'a oubliée.

Un fou à côté de moi débite sa frustration. Il délire au café à une table voisine. Personne ne lui parle. Tous le tolèrent

Il n'est pas encore neuf heures et la journée sera longue 